\title{
IN VIVO CONTACT STRESSES AT THE RADIOCARPAL JOINT USING A FINITE ELEMENT METHOD OF THE COMPLETE WRIST JOINT
}

\author{
Magnus K. Gislason (1), Benedict Stansfield (1), David H. Nash (2) \\ 1. Bioengineering unit, University of Strathclyde, Glasgow, UK, 2. Department of Mechanical \\ Engineering, University of Strathclyde, Glasgow, UK
}

\begin{abstract}
Introduction
A small number of cadaveric studies have been carried out looking at the force transmission through the radiocarpal joint. Pressure sensitive films have been placed at the joint to estimate the contact stresses under loading of the wrist [Tencer 1988]. Measurements using pressure sensitive films are difficult to quantify and the invasive procedure of placing the films could potentially perturb the joints. Tencer et al reported average contact pressure of $3.2 \mathrm{MPa}$, with the cadaveric wrist loaded with a compressive load of $103 \mathrm{~N}$. Computational studies have also addressed this issue. In 2003 Carrigan et al created a finite element model of the wrist and reported contact stresses of 2.6 MPa at the distal end of the radius. The loading was $15 \mathrm{~N}$ compressive loading acting on the capitate. Under loading the wrist can take up large amount of force with values around 2 times bodyweight travelling through the joints [Chadwick \& Nicol 2000]. In this study subject specific finite element models were created of the whole wrist joint using measured biomechanical data to capture the forces acting on the wrist with the hand generating a maximum gripping force.
\end{abstract}

\section{Methods}

Three subjects took part in this study, all young and healthy adults. The kinetic and kinematic data were captured using five 6-degrees of freedom force transducers in conjunction with 8 camera Vicon system. The force transducers were mounted on a gripping tool which subjects squeezed, generating their maximum gripping force in a functionally neutral position. The forces measured were the external forces acting on the distal phalanges. The subjects were then taken for an MRI scan of their wrists. The scan resolution was 234x234 $\mu \mathrm{m}$ ranging from the distal end of the radius and ulna to the proximal third of the metacarpals. The slice thickness was $750 \mu \mathrm{m}$. The scans were imported into Mimics from Materialize where edge detection of the bones was carried out for the finite element analysis. The edges of the bones were identified for each slice and from the contours, three-dimensional objects of the carpus were created. The bones were meshed within Mimics and exported into Abaqus v.6.6-1 where the assembly of the model took place. The external forces were converted into joint contact forces using an inverse dynamic biomechanical model. Material properties of the bones were identified using the MRI scans and ligaments and tendons were modelled within Abaqus using non-linear spring elements.

\section{Results}

The models were run under the calculated threedimensional loading conditions acting on the metacarpals. Resultant joint contact forces on the metacarpals ranged from $1250 \mathrm{~N}-1850 \mathrm{~N}$. Stress plots were generated for the bones of the wrist (e.g. Fig. 1). Average contact stresses on the distal end of the radius are given in Table 1 as an example of model output.

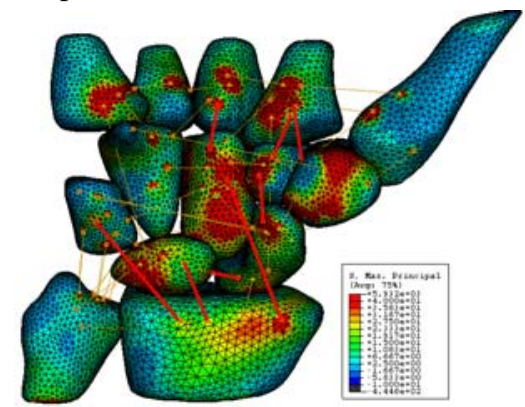

Figure 1: Stress contour plot of the whole wrist

\begin{tabular}{cccc}
\hline & Subject 1 & Subject 2 & Subject3 \\
\hline Radiolunate [MPa] & 7.0 & 5.6 & 23.4 \\
Radioscaphoid [MPa] & 8.0 & 5.7 & 15.3
\end{tabular}

Table 1: Average contact stresses on the distal end of the radius.

The results indicated that the in-vivo contact stresses were higher than had previously been reported through other computational models and cadaveric studies. Unexpected high stress values were seen for subject 3 . Analysis showed that by reducing the loading, the contact stresses became similar to the ones presented through cadaveric studies. This study has shown that the use of physiological loading conditions is essential in order to predict in vivo stresses in the carpus.

\section{References}

Tencer et al, J Orthop Res, Vol 6: 4:509-517, 1988. Carrigan et al, Annals of Biomed. Eng., Vol 31:718725, 2003.

Chadwick et al, J Biomech, Vol 33:591-600, 2000. 